\title{
Glutathione S-transferase polymorphisms may be associated with risk of oedematous severe childhood malnutrition
}

\author{
Kwesi G. Marshall ${ }^{1}$, Sharon Howell ${ }^{1}$, Marvin Reid $^{2}$, Asha Badaloo ${ }^{1}$, Martin Farrall ${ }^{3}$, Terrence Forrester ${ }^{1}$ \\ and Colin A. McKenzie ${ }^{1 *}$ \\ ${ }^{1}$ Tropical Metabolism Research Unit, University of the West Indies, Mona, Jamaica \\ ${ }^{2}$ Sickle Cell Unit, Tropical Medicine Research Institute, University of the West Indies, Mona, Jamaica \\ ${ }^{3}$ Department of Cardiovascular Medicine, University of Oxford, Wellcome Trust Centre for Human Genetics, Oxford, UK
}

(Received 16 August 2005 - Revised 30 January 2006 - Accepted 3 April 2006)

\begin{abstract}
It has been estimated that more than $50 \%$ of deaths before the age of 5 years have undernutrition as an underlying cause. Severe childhood malnutrition, an extreme form of undernutrition, occurs as oedematous and non-oedematous syndromes. The reasons why only some children develop oedematous severe childhood malnutrition (OSCM) have remained elusive, but the heterogeneity of clinical appearances among children from relatively homogeneous backgrounds suggests that interindividual variation in susceptibility to OSCM may exist. We investigated variants of four glutathione S-transferase (GST) genes in a retrospective study among subjects ( $n$ 136) previously admitted to the Tropical Metabolism Research Unit, Jamaica, for the treatment of either OSCM (cases) or non-oedematous severe childhood malnutrition (controls). We found that GSTP1 Val ${ }^{105}$ homozygotes were significantly more common among the cases (odds ratio (OR) $3 \cdot 5 ; 95 \%$ CI 1.1, 10.8). We also found an association of borderline significance between non-deletion GSTT1 genotypes (i.e. $+/+$ or $+/ 0)$ and OSCM (OR $2 \cdot 4 ; 95 \%$ CI $1 \cdot 0,5 \cdot 9)$. There was no significant association between OSCM and any of the other GST variants. These preliminary findings suggest that genetic variation within the GST superfamily may contribute to the risk of OSCM. Additional, larger data sets and studies of variants in other candidate genes are required in order to properly assess the true contribution, if any, of genetic variation to risk of OSCM. Such studies may improve our understanding of the causes of clinical heterogeneity in malnutrition.
\end{abstract}

Oedematous childhood malnutrition: GSTP1: GSTT1

Undernutrition in childhood is an important public health problem worldwide. It is estimated that more than 5.5 million deaths among children under the age of 5 years have undernutrition as an underlying cause (Caulfield et al. 2004; Bryce et al. 2005). Severe childhood malnutrition (SCM), with the highest mortality risk, includes clinical presentations that range from marked weight loss only, to marked weight loss plus dramatic hair and skin changes that are often associated with oedema. In many centres, however, only the degree of weight deficit and the presence or absence of oedema are routinely used to categorise these clinical syndromes (Lancet, 1970; World Health Organization, 1999, 2000).

Blood concentrations of GSH are considerably lower in oedematous SCM (OSCM) than in non-oedematous SCM (NOSCM), with little or no overlap between the two groups (Jackson, 1986a,b; Golden \& Ramdath, 1987). GSH is a tripeptide (glutamic acid-cysteine-glycine) of almost universal tissue distribution and is the major source of cellular low-molecular weight sulfhydryl $(-\mathrm{SH})$ groups, providing protection against attack by peroxides, reactive oxygen species and electrophilic xenobiotics (Wang \& Ballatori, 1998).
The observation of a low GSH level in kwashiorkor was important in the development of the free radical theory (Golden \& Ramdath, 1987), which proposes that the development of the features of OSCM is due to an imbalance between the generation of free radicals and their safe disposal.

The cause of low GSH levels in OSCM is uncertain. We have recently shown that GSH synthesis rates are lower in OSCM than NOSCM (Reid et al. 2000) and that cysteine supplementation increases in vivo erythrocyte GSH synthesis rates in OSCM (Badaloo et al. 2002). These observations suggest that a limiting availability of precursors such as cysteine may lead to reduced concentrations of GSH and may thus play a primary role in the pathogenesis of OSCM. It is possible that there may be alternative or additional causal explanations, however, as low levels of precursors may be a secondary phenomenon.

The proportion of patients suffering from OSCM varies by locale (Hendrickse, 1991; Waterlow, 1992). Within any particular locale, however, patients with OSCM appear to be similar to patients with NOSCM in terms of socio-economic status, nutritional history, rate of infection and many biochemical variables (Gopalan, 1970; Laditan \& Reeds,

Abbreviations: GST, glutathione S-transferase; NOSCM, non-oedematous severe childhood malnutrition; OR, odds ratio; OSCM, oedematous severe childhood malnutrition; SCM, severe childhood malnutrition.

* Corresponding author: Dr Colin A. McKenzie, fax +1 876977 0632, email colin.mckenzie02@uwimona.edu.jm 
1976; Christie et al. 1992; Waterlow, 1992). The very different clinical outcomes seen among children with similar environmental exposures suggests the possibility that there may be interindividual variation in the ability to handle these exposures. Such variation might explain why some children end up with OSCM (and low GSH levels) despite having very similar backgrounds to children with NOSCM.

There are several different isoforms of glutathione S-transferase (GST); these isoforms catalyse the irreversible conjugation of GSH to a wide variety of electrophilic compounds including organic hydroperoxides, unsaturated hydroxyalkenals and epoxides (Commandeur et al. 1995; Wang \& Ballatori, 1998). In SCM, in which there are both macronutrient and micronutrient deficiencies, many of the 'normal' antioxidant defences are likely to be compromised (Golden \& Ramdath, 1987), and there may thus be an increase in the relative importance of GST-mediated anti-oxidant defence. In addition, there are genetic variants, which alter the catalytic activity of several of the GST isoforms (Chen \& Board, 1987; Seidegård et al. 1988; Zimniak et al. 1994; Inskip et al. 1995). It is possible, therefore, that an inherited variation in GST activity might play a role in predisposition to oxidant damage and susceptibility to OSCM.

In the present paper, we report on a case-control study among Jamaican children previously admitted to hospital for the treatment of SCM. We explore associations between genetic variants in four enzymes of the GST superfamily and the risk of a diagnosis of OSCM. We also explore the relationships between urinary isoprostanes (a marker of in vivo lipid peroxidation), nutritional diagnosis and GST genotypes.

\section{Materials and methods}

\section{Participants}

The metabolic research ward of the Tropical Metabolism Research Unit, Faculty of Medical Sciences, University of the West Indies, is located at the University Hospital of the West Indies in the parish of St Andrew at the south-eastern end of Jamaica. The Tropical Metabolism Research Unit ward also serves as a tertiary referral centre for children with severe malnutrition from the entire island. Admission records for the ward were reviewed in order to generate a list of potential subjects.

The principal inclusion criterion was a diagnosis of SCM according to the Wellcome Classification (Lancet, 1970), that is, a diagnosis of marasmus $(<60 \%$ weight-for-age, no oedema), marasmic kwashiorkor ( $<60 \%$ weight-for-age with oedema) or kwashiorkor $(60-80 \%$ weight-for-age with oedema). Patients who had 'secondary malnutrition' (e.g. HIV seropositivity, congenital malformations, cerebral palsy) were excluded. A total of 691 patients met these criteria. Potential participants were also required to have had an address in Kingston (the capital city, whose limits are within the parish of St Andrew), St Andrew or St Catherine (a neighbouring parish to the west of St Andrew) and to have been discharged from the ward not more than 15 years previously. This additional criterion reduced the number of potentially eligible participants to 320 . The study was approved by the University Hospital of the West Indies/University of the West Indies Faculty of Medical
Sciences Ethics Committee, and written, informed consent was given by a parent or other adult guardian for each child.

At the end of the recruitment period, we had attempted to contact 256 former patients; 136 of whom were eventually recruited. Among these former patients, three were excluded after a review of their original admission data indicated that they were ineligible. There were several sets of siblings (including twins) among the former patients; for any set of siblings, only the child with the earliest date of admission was retained in the data set for analysis. If siblings were admitted to the ward on the same day, one child from the sibship was chosen at random. Thus, the final data set contained 124 former patients. Of the 120 former patients who were not recruited, we were unable to trace fortynine. We contacted fifty-three who were not successfully recruited ('not interested', 'appointment dates/times not convenient'). Eleven were reported to have moved or migrated, and seven were reported to be dead.

\section{Anthropometry and laboratory assays}

Height and weight measurements were made on all subjects. All measurements were carried out at the Tropical Metabolism Research Unit using standardised procedures (Cooper et al. 1997). Height-for-age and BMI $z$ scores were calculated using the 2000 CDC charts option in Epi Info (version 3.3.2; Centers for Disease Control and Prevention, Atlanta, GA, USA).

DNA was extracted from blood samples using a phenol/ chloroform protocol (Sambrook et al. 1989). Genotypes of biallelic polymorphisms within four GST enzymes $(\theta 1$, GSTT1; $\mu$ 1, GSTM1; $\mu$ 3, GSTM3; $\pi$ 1, GSTP1) were determined by agarose gel electrophoresis of either PCR products or restriction enzyme digests of PCR products followed by ethidium staining of the gels. All genotyping was performed according to previously published protocols with only minor modifications (Brockmöller et al. 1993; Pemble et al. 1994; Inskip et al. 1995; Harries et al. 1997).

For both GSTT1 and GSTM1, the polymorphism that was typed is caused by a large deletion in which the null genotype $(0 / 0)$ is associated with absence of enzyme activity. In the present PCR-based assay, 0/+ heterozygotes were not distinguishable from $+/+$ homozygotes. Thus, in the analysis for these genes, only 'null' (i.e. 0/0) and 'positive' (i.e. 0/+ and $+/+$ combined) genotypes were considered. For the biallelic polymorphisms in GSTP1 and GSTM3, the three possible genotypes could be detected. The polymorphism in GSTP1 is caused by a point mutation; the variant allele codes for valine rather than isoleucine at amino acid 105 and is associated with changes in enzyme activity towards different substrates (Hayes \& Strange, 2000). The polymorphism in GSTM3 results from a $3 \mathrm{bp}$ insertion/deletion in intron 6 and thus does not change the primary sequence of the protein product. The deletion variant, however, harbours a recognition motif for a negative transcription factor and could be a marker of reduced GSTM3 activity (Inskip et al. 1995).

A spot urine sample was collected from all subjects. The concentration of urinary isoprostanes (principally 8-iso-prostaglandin $\mathrm{F}_{2 \alpha}$ ), a marker of in vivo lipid peroxidation (Halliwell \& Gutteridge, 1999; Pratico et al. 2004), was estimated using an ELISA method that has been implemented in a commercially available kit (Oxford Biomedical Research, Oxford, MI, USA). Urinary creatinine concentrations were measured 
on an Abbott Alcyon 300i autoanalyser (Abbott Laboratories, Abbott Park, IL, USA). Concentrations of isoprostanes are expressed as $\mathrm{ng} / \mathrm{mmol}$ creatinine. Samples from a large urine pool were included in each analytical run, yielding a $\mathrm{CV}$ of $12 \%$ for urinary isoprostanes.

\section{Statistical methods}

The principal aim was to determine whether there were differences in either genotype or allele frequencies between cases and controls. The strength of this association is expressed in terms of the odds ratio (OR) and $95 \% \mathrm{CI}$ for a previous diagnosis of OSCM. A secondary aim was to explore whether there were between-group (cases $v$. controls) or between-genotype differences in the level of in vivo lipid peroxidation as estimated by concentrations of urinary isoprostanes. Between-group differences were analysed using $\chi^{2}$ tests or Student's $t$ tests as appropriate. Linear regression models were used to determine whether there were significant associations between urinary isoprostanes, diagnosis and genotype. The significance of terms in the regression models was tested using a manual, stepwise, backward elimination procedure; pairs of models were evaluated with likelihood-ratio tests. Differences in $-2 \times \log _{\mathrm{e}}$ (likelihood) are approximated by a $\chi^{2}$ distribution with degrees of freedom equal to the difference in the number of parameters fitted in each model. All models were fitted using Stata 8 software (Stata Corporation, College Station, TX, USA).

\section{Results}

Tests of association between glutathione S-transferase genotypes and OSCM

In the final data set, seventy-four participants had been previously diagnosed as having OSCM (cases) and fifty participants were diagnosed as having NOSCM (controls). The mean age on admission was $15 \cdot 1$ (SD 21.5) months, and the mean elapsed time since admission was 7.6 (SD 4.5) years. The proportion of males among the cases was greater than among the controls (Table 1), but this difference was not significant $\left(\chi^{2} 0 \cdot 75,1 \mathrm{df}, P=0 \cdot 39\right)$.

The relationship between polymorphisms in different GST genes and the risk of having a previous diagnosis of OSCM is shown in Table 2. For GSTP1, there was a significant association between genotype and OSCM: subjects who were homozygous for the valine ${ }^{105}$ allele were more likely to have a previous diagnosis of OSCM (OR 3.48, $95 \%$ CI $1 \cdot 1,10 \cdot 8, P=0.0213$ ) when compared with isoleucine ${ }^{105}$ homozygotes. Heterozygotes were also more likely to have a previous diagnosis of OSCM when compared with isoleucine ${ }^{105}$ homozygotes (OR 2.25, $95 \%$ CI 0.90, 5.66), but this increased risk was not significant at the $5 \%$ level. A score test for trend indicated that the risk of OSCM increased significantly with the number of copies of the valine ${ }^{105}$ allele $\left(\chi^{2} 5 \cdot 89,1 \mathrm{df}, P=0 \cdot 015\right)$ and provided evidence of a 'dose-response effect'; unconditional logistic regression under an additive model gave an OR for OSCM of $1.92(95 \%$ CI $1.13,3.26)$ for each additional valine ${ }^{105}$ allele.

There was an association between GSTT1 genotype and OSCM: there was a larger proportion of subjects with the GSTT1 + genotype (i.e. $+/+$ or $+/ 0$ ) among the cases than among the controls $(33.3 \% v .17 \cdot 1 \%)$, a difference that was just significant at the $5 \%$ level $\left(\chi^{2} 4 \cdot 12,1 \mathrm{df}\right.$, $P=0.042$ ). The OR for a previous diagnosis of OSCM was $2.44(95 \%$ CI $1.00,5 \cdot 88)$ among subjects with the + genotype compared with subjects whose genotype was $0 / 0$.

For GSTM3, there was no evidence of a significant association between genotype and a previous diagnosis of OSCM. Insertion/deletion heterozygotes appeared to have a lower risk of a previous diagnosis of OSCM (OR 0.81, $95 \%$ CI 0.38 , 1.75), and the infrequent insertion/insertion homozygotes appeared to have a higher risk of OSCM (OR 2.67, $95 \% \mathrm{CI}$ $0.50,14.4)$ when compared with deletion/deletion homozygotes. In neither case, however, were the risk ratios significantly different from 1, and there was no significant change in the risk of OSCM with increasing numbers of copies of the insertion allele ( $\chi^{2}$ for trend $0 \cdot 24,1 \mathrm{df}, P=0 \cdot 62$ ).

For GSTM1, there was also no significant association with OSCM. The proportion of subjects with the + genotype among the cases was somewhat higher than that among the controls $(36.0 \%$ v. $25.4 \%)$, but this difference was not significant $\left(\chi^{2} 1 \cdot 59,1 \mathrm{df}, P=0 \cdot 207\right)$. The OR for OSCM among subjects with the + genotype was $1.67(95 \%$ CI $0.75,3.70)$ when compared with subjects whose genotype was $0 / 0$.

\section{Tests of between-group and between-genotype differences in urinary isoprostane level}

We also examined the determinants of urinary isoprostanes among the cases and controls; we were particularly interested

Table 1. Current age, weight, height, BMl and urinary isoprostane levels for subjects with previous diagnoses of oedematous (cases) and non-oedematous (controls) severe childhood malnutrition

(Values are means and standard deviations)

\begin{tabular}{|c|c|c|c|c|c|}
\hline & \multicolumn{2}{|c|}{ Cases } & \multicolumn{2}{|c|}{ Controls } & \multirow[b]{2}{*}{$P$ values ${ }^{*}$} \\
\hline & Mean & SD & Mean & SD & \\
\hline Age (years) & 7.5 & 4.9 & $9 \cdot 6^{*}$ & 3.9 & 0.010 \\
\hline Weight (kg) & $25 \cdot 3$ & $16 \cdot 9$ & $28 \cdot 1$ & $13 \cdot 6$ & NS \\
\hline Height (m) & $118 \cdot 1$ & 30.9 & $129 \cdot 4^{*}$ & $22 \cdot 8$ & 0.023 \\
\hline BMI $\left(\mathrm{kg} / \mathrm{m}^{2}\right)$ & $16 \cdot 5$ & $3 \cdot 0$ & $15 \cdot 7$ & 2.5 & NSt \\
\hline
\end{tabular}

${ }^{*} P$ values are two-sided and are derived from the unpaired $t$ test or, in the case of the percentage of males in each group, from a $\chi^{2}$ test with $1 \mathrm{df}$. $\dagger P>0.05$.

For details of subjects and procedures, see p. 244. 
Table 2. Risk ratios for a previous diagnosis of oedematous severe childhood malnutrition associated with genetic polymorphisms of genes encoding glutathione S-transferase (GST)

\begin{tabular}{|c|c|c|c|c|}
\hline & Cases & Controls & Odds ratio & $95 \% \mathrm{Cl}$ \\
\hline \multicolumn{5}{|l|}{ GSTP1 } \\
\hline Ile/lle & 17 & 23 & 1.00 & \\
\hline Ile/Val & 25 & 15 & $2 \cdot 25$ & $0.90,5.66$ \\
\hline $\mathrm{Val} / \mathrm{Val}$ & 18 & 7 & 3.48 & $1 \cdot 12,10 \cdot 78$ \\
\hline \multicolumn{5}{|l|}{ GSTT1 } \\
\hline $0 / 0$ & 12 & 16 & 1.00 & \\
\hline$+1+$ or +10 & 58 & 32 & $2 \cdot 44$ & $1 \cdot 00,5 \cdot 88$ \\
\hline \multicolumn{5}{|l|}{ GSTM3 } \\
\hline Deletion/deletion & 30 & 20 & 1.00 & \\
\hline Insertion/deletion & 33 & 27 & 0.81 & $0.38,1.75$ \\
\hline Insertion/insertion & 8 & 2 & $2 \cdot 67$ & $0.50,14 \cdot 36$ \\
\hline \multicolumn{5}{|l|}{ GSTM1 } \\
\hline $0 / 0$ & 18 & 18 & 1.00 & \\
\hline$+1+$ or $+/ 0$ & 53 & 32 & 1.67 & $0.75,3 \cdot 70$ \\
\hline
\end{tabular}

For details of subjects and procedures, see p. 244.

in testing whether there were significant between-group or between-genotype differences in isoprostanes in this group of participants with documented histories of SCM who were not severely malnourished (mean height-for-age $z$ score -0.85 (SD 1.8), mean BMI $z$ score -0.75 (SD 1.1)) at the time they were studied; a significant association with genotype would suggest that inherited variation might contribute to the differences between OSCM and non-OSCM. Table 1 provides data on anthropometric variables and urinary isoprostanes for cases and controls. In a preliminary survey of the differences between the two groups, the level of urinary isoprostanes was found to be lower among controls than the cases, and this difference was significant $(t-2 \cdot 5, P=0 \cdot 013)$. The controls were older, taller and heavier than the cases; the differences between groups for age $(t 2 \cdot 6, P=0.010)$ and height $(t 2 \cdot 2$, $P=0.033)$ were significant, but those for weight and BMI were not $(P>0.05)$.

In order to determine whether previous SCM status or any of the GST genotypes was associated with the level of urinary isoprostanes after adjustment for the age and anthropometric differences between the case and control groups, we fitted multivariable linear regression models that included terms for OSCM (i.e. either case or control), gender, age, age ${ }^{2}$ and height, and also for the different GST genotypes as predictors. There was significant heteroscedasticity of urinary isoprostanes, so the regression models were fitted to the isoprostanes data after $\log$ transformation. The regression coefficients and associated standard errors for OSCM and for the GST genotypes were estimated from the most general model and are given in Table 3. Each coefficient represents the effect of that variable on urinary isoprostanes after adjustment for the effects of the other variables in the model. In this model, the previous nutritional state of a subject was not a significant predictor of current level of urinary isoprostanes $(B 20 \cdot 2, P>0.05)$, nor were any of the GST genotypes ( $P>0.05$ for all). The only terms that had a significant influence on model fit were age, age ${ }^{2}$ and height.

As an additional exploration, ANOVA models were fitted to the isoprostane data, including a term for diagnosis that had three levels (kwashiorkor, marasmic kwashiorkor, marasmus), rather than the two levels of 'cases' and 'controls'. This was done to determine whether there were differences between the two types of OSCM. The variance due to diagnosis defined in this way was, however, small and not significant both with and without the inclusion of the covariates included in the linear regression models (data not shown).

\section{Discussion}

GSH plays an important role in the body's antioxidant defences, and low GSH levels indicate that these are impaired. It is not entirely certain, however, how GSH becomes depleted, nor is it clear why low GSH levels should be found in OSCM and not NOSCM. Recent data from Malawi, where a double-blind randomised trial of antioxidant supplementation failed to demonstrate a significant reduction in risk of kwashiorkor (Ciliberto et al. 2005), further emphasise these uncertainties surrounding the pathogenesis of OSCM. The existence of interindividual variation in the ability to handle the stresses imposed by malnutrition might help to explain heterogeneous clinical outcomes in the face of apparently homogeneous environments. The hypothesis explored in this paper is that persons who had developed OSCM and its associated increased levels of oxidant damage would have different genotype and allele frequencies for genetic variants of different GST isoforms when compared with those who had NOSCM.

In the present retrospective study, we have examined the association between OSCM and the functional or potentially functional variants that have been described (Chen \& Board, 1987; Seidegård et al. 1988; Zimniak et al. 1994; Inskip et al. 1995) in four genes of the GST superfamily; the use of functional variants instead of 'anonymous' or

Table 3. Coefficients $(B)$ and standard errors for the regression of urinary isoprostanes on age, age ${ }^{2}$, height, previous nutritional status (oedematous severe childhood malnutrition) and glutathione S-transferase (GST) genotype

\begin{tabular}{lccc}
\hline Variable & $B$ & SE & $P$ value* \\
\hline Oedematous severe childhood malnutrition & 20.2 & 23.3 & 0.39 \\
GSTM1 & -51.5 & 23.7 & 0.14 \\
GSTT1 & -0.45 & 26.8 & 0.60 \\
GSTP1 & -14.9 & 14.1 & 0.12 \\
GSTM3 & -12.0 & 18.0 & 0.20 \\
\hline
\end{tabular}

* Estimated from the ratio of the likelihood of the general model to that of a restricted model.

For details of subjects and procedures, see p. 244. 
non-functional genetic markers potentially makes the task of establishing the biological connection between DNA sequence variation and outcomes simpler. We found that there was a significant association between the valine ${ }^{105}$ variant of GSTP1 and risk of OSCM: subjects who were homozygous for the valine ${ }^{105}$ allele were almost 3.5-fold more likely to have had a previous diagnosis of OSCM $(P=0.02)$. We also found that subjects with the GSTT1 + genotype (i.e. $+/ 0$ or $+/+$ ) had an approximately 2.4-fold higher risk of OSCM, which was of borderline significance $(P=0 \cdot 04)$. In the case of GSTM1 and GSTM3, no significant associations were found. We did note, however, that GSTM1 +, like GSTT1 +, was associated with a higher risk of OSCM (approximately 1.7-fold), even though the increased risk was not significant $(P=0 \cdot 21)$. These preliminary data provide some support for the idea that genetic variation in GST may contribute to risk of OSCM.

Based on the roles of GST in detoxifying harmful xenobiotics (Commandeur et al. 1995; Wang \& Ballatori, 1998; Hayes \& Strange, 2000; Rinaldi et al. 2002), it might have been expected that GST variants with reduced activity would confer an increased risk of OSCM, but this does not appear to be the case in the present study. One possibility is that the variants that appear to be associated with an increased risk of OSCM may actually catalyse the bioactivation or toxification of some endogenous or exogenous ligand; this has been described for substrates of GSTT1 and GSTP1 (Commandeur et al. 1995; Sherratt et al. 1997). An alternative possibility, which is plausible for the significant association observed for the GSTP1 variant, is that the associated variant does actually have decreased activity against some (unknown) reactive substrate. The valine ${ }^{105}$ GSTP1 variant, which has increased activity against anti-diol epoxides of benzo(a)pyrene, has reduced activity against chlorodinitrobenzene and anti-diol epoxides of benzo[g]chrysene compared with isoleucine $^{105}$ (Hayes \& Strange, 2000). Although either of these proposed explanations would be expected to result in a higher risk of oxidant damage to cell constituents, the biological basis for the observed associations remains to be established.

Erythrocyte GST activity against chlorodinitrobenzene has been reported to be increased in children with OSCM or NOSCM compared with children without (Ramdath \& Golden, 1988, 1993). These reports underscore the increased relative importance of GST pathways in SCM but provide little, if any, information about the influence of genetic variation on risk of OSCM. Chlorodinitrobenzene is a relatively non-specific substrate for GST, with only a small difference in activity between the most and least effective isoenzymes (van Ommen et al. 1990). Studies using chlorodinitrobenzene would thus be expected to have low power to detect the contribution of genetic variants to between-group differences in erythrocyte GST activity. Of potentially greater importance, in any event, would be measures of the impact of genetic variation in GST activity on metabolic processes in, for example, the vascular endothelium or liver.

It is also possible, even if there are biologically plausible explanations, that the differential survival of patients with low-activity alleles or some other type of selection bias, or type I error, might account for the observed associations. For example, the in-hospital mortality of patients with OSCM has been reported to be higher than that of patients with NOSCM (Schofield \& Ashworth, 1996), but the low overall mortality (approximately $7 \%$ ) at the Tropical Metabolism Research Unit (Cooper \& Smartt, 1996) suggests that the magnitude of this particular bias is likely to be small. We also found that there were no significant between-group or between-genotype differences in the excretion of urinary isoprostanes. This finding is consistent with the view that the effect of variant GST on lipid peroxidation would be expected to be small or negligible among persons who have been restored to normal nutritional status. It seems unlikely, therefore, that there would be an association between GST genotype and survival after discharge from hospital. Ultimately, however, the possible effects of biases and chance will need to be addressed by attempting to replicate the present findings.

Studies of GSH that utilise stable isotope methods in a small number of research participants provide important information about metabolic adaptations in SCM (Reid \& Jahoor, 2000, 2001). There remain difficulties, however, in distinguishing primary from secondary phenomena. The study of genetic determinants may be useful in this regard; associations between outcomes and functional genetic polymorphisms, in which the polymorphisms mimic a proposed exposure, may provide important clues about causation and are not likely to be susceptible to 'reverse causation' (Youngman et al. 2000; Davey Smith \& Ebrahim, 2003). Furthermore, the inferences derived from genetic studies are not restricted to easily available cells coding information about isoforms expressed in inaccessible tissues is available from any DNA-containing cell.

It has only recently been demonstrated that outcomes in infectious (i.e. 'environmental') diseases such as Plasmodium falciparum malaria, tuberculosis and HIV/AIDS are influenced by variants in a range of genes (Segal \& Hill, 2003). The results of the present small study provide some support for the idea that genetic variation might contribute to the clinical picture of OSCM. Larger, retrospective and prospective studies of genetic variants of GST and other candidate genes, in Jamaica and elsewhere, will be required in order to confirm our preliminary findings. Such studies may provide insights that could complement other approaches to the understanding of OSCM.

\section{Acknowledgements}

Principally, we are grateful to the former patients of the Tropical Metabolism Research Unit, University of the West Indies, Jamaica, who participated in this study. We are also grateful for the assistance of Dr Michelle Williams-Robinson and Dr Karen Phillips, Mr Bentley Chambers, Sister Ethlyn Henry and the nursing staff of the Tropical Metabolism Research Unit, Ms Joan Patterson and Mr Gary Gauntlett in carrying out this retrospective study. These data were presented in part at the 49th Annual Conference of the Caribbean Health Research Council, Grenada, West Indies, April 2004. This study was supported by a grant from the Rhodes Trust to C. A. M.

\section{References}

Anon (1970) Classification of infantile malnutrition. Lancet ii, $302-303$. 
Badaloo A, Reid M, Forrester T, Heird WC \& Jahoor F (2002) Cysteine supplementation improves the erythrocyte glutathione synthesis rate in children with severe edematous malnutrition. Am J Clin Nutr 76, 646-652.

Brockmöller J, Kerb R, Drakoulis N, Nitz M \& Roots I (1993) Genotype and phenotype of glutathione S-transferase class $\mathrm{m}$ isoenzymes $\mathrm{m}$ and $\mathrm{y}$ in lung cancer patients and controls. Cancer Res 53, 1004-1011.

Bryce J, Boschi-Pinto C, Shibuya K \& Black RE (2005) WHO estimates of the causes of death in children. Lancet $\mathbf{3 6 5}$, $1147-1152$.

Caulfield LE, de Onis M, Blossner M \& Black RE (2004) Undernutrition as an underlying cause of child deaths associated with diarrhea, pneumonia, malaria, and measles. Am J Clin Nutr 80, 193-198.

Chen LZ \& Board PG (1987) Hgi AI restriction fragment length polymorphism at the human glutathione S-transferase 2 locus. Nucleic Acids Res 15, 6306

Christie CDC, Heikens GT \& Golden MHN (1992) Coagulase-negative staphylococcal bacteremia in severely malnourished Jamaican children. Pediatr Infect Dis J 11, 1030-1036.

Ciliberto H, Ciliberto M, Briend A, Ashorn P, Bier D \& Manary M (2005) Antioxidant supplementation for the prevention of kwashiorkor in Malawian children: randomised, double blind, placebo controlled trial. Br Med J 330, 1109.

Commandeur JM, Stijntjes GJ \& Vermeulen NPE (1995) Enzymes and transport systems involved in the formation and disposition of glutathione S-conjugates. Role in bioactivation and detoxication mechanisms of xenobiotics. Pharmacol Rev 47, 271-330.

Cooper ES \& Smartt V (1996) Trends in admission and case fatality for acute malnutrition. West Indian Med J 45, 22-24.

Cooper R, Rotimi C, Ataman S, et al. (1997) The prevalence of hypertension in seven populations of West African origin. Am J Public Health 87, 160-168.

Davey Smith G \& Ebrahim S (2003) 'Mendelian randomization': can genetic epidemiology contribute to understanding environmental determinants of disease? Int J Epidemiol 32, 1-22.

Golden MHN \& Ramdath D (1987) Free radicals in the pathogenesis of kwashiorkor. Proc Nutr Soc 46, 53-68.

Gopalan C (1970) Some recent studies in the Nutrition Research Laboratories, Hyderabad. Am J Clin Nutr 23, 35-51.

Halliwell B \& Gutteridge JMC (1999) Free Radicals in Biology and Medicine, 3rd ed. Oxford: Oxford University Press.

Harries LW, Stubbins MJ, Forman D, Howard GCW \& Wolf CR (1997) Identification of genetic polymorphisms at the glutathione S-transferase Pi locus and association with susceptibility to bladder, testicular and prostate cancer. Carcinogenesis 18, 641-644.

Hayes JD \& Strange RC (2000) Glutathione S-transferase polymorphisms and their biological consequences. Pharmacology 61, $154-166$.

Hendrickse RG (1991) Protein-energy malnutrition. In Paediatrics in the Tropics, pp. 119-131 [RG Hendrickse, DGD Barr and TS Matthews, editors]. Oxford: Blackwell Scientific Publications.

Inskip A, Elexperu-Camiruaga J, Buxton N, et al. (1995) Identification of polymorphism at the glutathione S-transferase, GSTM3 locus: evidence for linkage with GSTM1*A. Biochem J 312, 713-716.

Jackson AA (1986a) Blood glutathione in severe malnutrition in childhood. Trans R Soc Trop Med Hyg 80, 911-913.

Jackson AA (1986b) Severe undernutrition in Jamaica. Kwashiorkor and marasmus: the disease of the weanling. Acta Paediatr Scand 323, Suppl., 43-51.

Laditan AAO \& Reeds PJ (1976) A study of the age of onset, diet and the importance of infection in the pattern of severe protein-energy malnutrition in Ibadan, Nigeria. Br J Nutr 36, 411-419.

Pemble S, Schroeder KR, Spencer SR, Meyer DJ, Hallier E, Bolt HM, Ketterer B \& Taylor JB (1994) Human glutathione S-transferase theta (GSTT1): cDNA cloning and the characterization of a genetic polymorphism. Biochem J 300, 271-276.
Pratico D, Rokach J, Lawson J \& FitzGerald GA (2004) F2-isoprostanes as indices of lipid peroxidation in inflammatory diseases. Chem Phys Lipids 128, 165-171.

Ramdath DD \& Golden MHN (1988) Elevated glutathione S-transferase activity in erythrocytes from malnourished children. In Medical, Biochemical and Chemical Aspects of Free Radicals: Proceedings of the 4th Biennial Meeting of the Society for Free Radical Research, 9-13 April 1988, pp. 567-570 [O Hayaishi, E Nike, M Kondo and T Yoshikawa, editors]. Amsterdam: Elsevier Science Publishers BV.

Ramdath DD \& Golden MHN (1993) Elevated glutathione S-transferase activity in erythrocytes from malnourished children. Eur J Clin Nutr 47, 658-665.

Reid M, Badaloo A, Forrester T, Morlese JF, Frazer M, Heird WC \& Jahoor F (2000) In vivo rates of erythrocyte glutathione synthesis in children with severe protein-energy malnutrition. Am J Physiol Endocrinol Metab 278, E405-E412.

Reid M \& Jahoor F (2000) Methods for measuring glutathione concentration and rate of synthesis. Curr Opin Clin Nutr Metab Care 3, 385-390.

Reid M \& Jahoor F (2001) Glutathione in disease. Curr Opin Clin Nutr Metab Care 4, 65-71.

Rinaldi R, Eliasson E, Swedmark S \& Morgenstern R (2002) Reactive intermediates and the dynamics of glutathione transferases. Drug Metab Dispos 30, 1053-1058.

Sambrook J, Fritsch EF \& Maniatis T (1989) Molecular Cloning: A Laboratory Manual, 2nd ed. Cold Spring Harbor, NY: Cold Spring Harbor Laboratory.

Schofield C \& Ashworth A (1996) Why have mortality rates for severe malnutrition remained so high? Bull World Health Organ 74, 223-229.

Segal S \& Hill AV (2003) Genetic susceptibility to infectious disease. Trends Microbiol 11, 445-448.

Seidegård J, Vorachek WR, Pero RW \& Pearson WR (1988) Hereditary differences in the expression of the human glutathione transferase active on trans-stilbene oxide are due to a gene deletion. Proc Natl Acad Sci USA 85, 7293-7297.

Sherratt PJ, Pulford DJ, Harrison DJ, Green T \& Hayes JD (1997) Evidence that human class theta glutathione S-transferase T1-1 can catalyse the activation of dichloromethane, a liver and lung carcinogen in the mouse. Comparison of the tissue distribution of GST T1-1 with that of classes alpha, mu and pi GST in human. Biochem J 326, Part 3, 837-846.

van Ommen B, Bogaards JJ, Peters WHM, Blaauboer B \& van Bladeren PJ (1990) Quantification of human hepatic glutathione S-transferase. Biochem J 269, 609-613.

Wang W \& Ballatori N (1998) Endogenous glutathione conjugates: occurrence and biological functions. Pharmacol Rev 5-0, $335-355$.

Waterlow JC (1992) Protein Energy Malnutrition, 2nd ed. London: Edward Arnold.

World Health Organization (1999) Management of Severe Malnutrition: A Manual for Physicians and Other Senior Health Workers. Geneva: World Health Organization.

World Health Organization (2000) Severe malnutrition. In Management of the Child with a Serious Infection or Severe Malnutrition. pp. 80-91. Geneva: World Health Organization.

Youngman LD, Keavney BD, Palmer A, Parish S, Clark S, Danesh J, Delepine M, Lathrop M, Peto R \& Collins R (2000) Plasma fibrinogen and fibrinogen genotypes in 4685 cases of myocardial infarction and in 6002 controls: test of causality by 'Mendelian randomization'. Circulation 102, 31-32.

Zimniak P, Nanduri B, Pikula S, Bandorowiez-Pikula J, Singhal SS, Srivastava SK, Awasthi S \& Awasthi YC (1994) Naturally-occurring human glutathione S-transferase GSTP1-1 isoforms with isoleucine and valine in position 104 differ in enzymatic properties. Eur J Biochem 244, 893-899. 